\title{
Modeling Planar Fluxgate Structures
}

\author{
Michael Ortner ${ }^{1,2, *}$, Neosha Navaei ${ }^{2}$ and Martin Lenzhofer ${ }^{1}$ \\ 1 Carinthian Tech Research AG, 9500 Villach,Austria; martin.lenzhofer@ctr.at \\ 2 Carinthia University of Applied Sciences, 9500 Villach, Austria; Niosha.navaie@gmail.com \\ * Correspondence: michael.ortner@ctr.at; Tel.: +43-4242-56000219 \\ † Presented at the Eurosensors 2018 Conference, Graz, Austria, 9-12 September 2018. \\ Published: 10 December 2018
}

\begin{abstract}
Planar fluxgate structures have been the focus of multiple experimental studies. However, theoretical treatises are still limited to the classical models that describe 3D structures. In this paper we derive an effective fluxgate equation for planar systems, dealing with strong stray fields and direct coupling, and show the stability and applicability of the Vacquier implementation. To support the theoretical model, FEM simulations are performed that also provide means of layouting planar fluxgates by pure magnetostatic simulation.
\end{abstract}

Keywords: magnetic sensors; planar fluxgate; modeling and simulation

\section{Introduction}

Fluxgate sensors are a type of vector magnetometer that originate in the first half of the $20^{\text {th }}$ century. They feature a wide range of resolutions spanning from $\mathrm{pT}$ to $\mathrm{mT}$ [1] and are the most sensitive type of magnetic field sensor available that does not require cryogenic cooling or similar extensive experimental setups. However, despite their advantages fluxgates have one major flaw: They are bulky with typical sensor head dimensions in the $\mathrm{cm}$ range as their sensitivity is directly proportional to the sensor size through the area $A$ of the core cross section and number of coil windings $N$, [2]. While miniature fluxgate sensors were realized by micro fabrication [3] and using integrated circuit technology [4] they cannot compete with state of the art Hall- or XMR technology. In addition to 3D structures, planar fluxgate layouts were proposed in the 1990s, [5]. They have two advantages over their 3D counterparts: Fabrication is easy using cheap and available PCB technology [6] and, despite their size, the planar form factor makes them potentially suitable for system integration. Furthermore, recent advances with asymmetric double core structures massively reduce the supply current while improving on sensitivity [7].

While a lot of experimental work was done on planar fluxgates, theoretical descriptions are still limited to the original works on 3D structures $[2,8]$. An extension to planar layouts was never attempted, possibly because an analytical solution seems difficult for such geometries. Some specific structures were studied numerically using the finite element method (FEM) [6]. In this work we study an extension of the fluxgate equation to planar systems and discuss additional effects that must be considered. The theoretical model is then supported by FEM simulation, aiming to advocate on planar fluxgate layout by magnetostatic simulation.

\section{Effective Model in Planar Geometries}

The fundamental idea behind the fluxgate mechanism is to use the non-linearity of the material magnetization characteristics $\mu(H)=\partial H B(H)$ of a known (soft) ferromagnetic core as an absolute marker to determine the value of a static external magnetic field $H_{0}$. This is realized by driving the core periodically into saturation and to determine the position of the characteristic non-linearity (bend 
in magnetization curve when reaching saturation) from the voltage output signal $V(t)$ of a coupled inductive element, see Figure $1 \mathrm{a}$. When $H_{0}$ is in superposition with a periodic excitation field $H_{e x}(t)$, the position of the bend is shifted that can be observed in the voltageoutput,

$$
V(t)=-\dot{\Phi}(t)=-N A \mu(t) \dot{H}_{e x}(t)-N A \dot{\mu}(t) H_{0}+O\left(H_{0}^{2}\right)
$$

a)

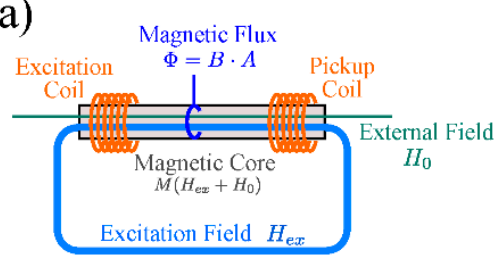

b)

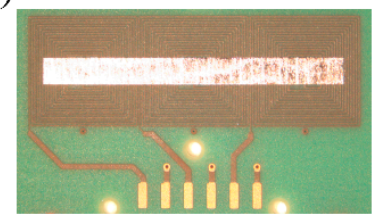

c)

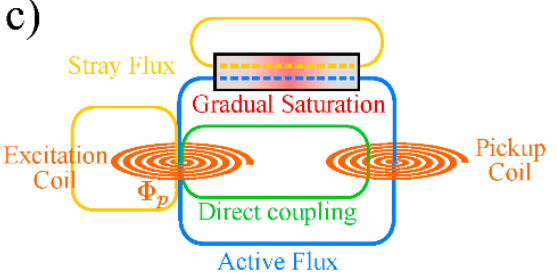

Figure 1. (a) Sketch of fluxgate mechanism, (b) PCB based sensor, (c) Additional effects in planar layouts.

Here $B\left(H_{e x}+H_{0}\right)$ in Faraday's law was expanded for small $H_{0}$ as proposed in [2]. The first term in this equation describes the classical induction which causes interference in the fluxgate, and the second term describes the fluxgate effect. A similar equation is given in [1].

The elegant Vacquier setup comprises two parallel, symmetric cores that carry opposing excitation fields of similar amplitude but aligned external fields. In this case two different voltages are obtained from (1) for the the two cores with $H=H_{e x} \pm H 0$. The voltages $V_{1}$ and $V_{2}$ are summed up so that the inductive terms cancel and the classical, basic fluxgate equation remains,

$$
V_{12}(t) \approx 2 N A H_{0} \dot{\mu}(t) \text {. }
$$

There are several ways to determine $H_{1}$ from the voltage output $V_{12}$. The most common and stable method is to detect the peak of the second harmonic in the Fourier transform of the output signal [1]. It follows from (2) that $V_{12}$ is non-zero only if $\mu$ is driven into the non-linear regime as $\dot{\mu}=$ $\mu^{\prime} \dot{B}$. The fluxgate mechanism thus requires $\max \left(H_{e x}(t)\right)>H_{S}$, where $H_{S}$ is a measure of the material saturation. In other words, the fluxgate works only if the core is driven into (deep) saturation each cycle. Sharp material magnetization bends and high frequencies increase $\mu$ and subsequently the devise sensitivity.

Derivation of Equations (1) and (2) are based on the idea that excitation and pickup coils fully enclose the core. The total magnetic flux in the magnetic circuit passes through the core and all coils and is homogeneously distributed, $\Phi(t)=A \cdot B(t)$. The magnetic induction is therefore directly related to the magnetic field through the material permeability, $B(t)=\mu(H(t)) H(t)$.

Such assumptions can be realistic when dealing with 3D setups and toroidal cores with homogeneously wound coils [8]. In true planar setups, however, this is not the case. Typically, such fluxgates are made up of adjacent (multilayer) spiral coils while the core is a thin stripe on top $[5,6]$. An example is shown in Figure $1 \mathrm{~b}$ where a PCB based planar structure, similar to the one studied in [7], is depicted. When modeling such planar geometries the coupling between excitation and pickup coils becomes more complicated. The idea is that the total flux $\Phi$ tot generated by the excitation coil and the magnetized core splits up into three parts $\Phi_{\mathrm{fg}}, \Phi_{\mathrm{S}}$ and $\Phi_{\mathrm{dc}}$ that must all be treated separately:

1 The active flux $\Phi_{\mathrm{fg}}$ which is responsible for the fluxgate effect. This is the part of the total flux that passes through the core and subsequently through the pickup coil. The inhomogeneous excitation field results in gradual saturation of the core and a smeared out saturation behavior.

2 Stray flux $\Phi_{\mathrm{S}}$ that does not contribute to the magnetic circuit. Stray flux is a result of the unnatural geometry of the system that is not easily followed by the magnetic field. It has no influence on the signal in the pickup coil, but results in a reduced efficiency of the system as more excitation current is required to generate the same amount of active flux. 
3 Direct coupling between the coils through $\Phi_{\mathrm{dc}}$. This is another result of the planar geometry, specifically between coil regions that are not located directly below the core. This effect results in a further loss of efficiency but also generates an additional, unwanted voltage output signal in the pickup coil.

For improved understanding these effects are outlined in Figure 1c. The voltage induced by the $i^{\prime}$ th winding of surface $A_{i}$ in the pickup coil is then given by

$$
V_{i}(t)=-\dot{\Phi}_{\mathrm{fg}, i}-\dot{\Phi}_{\mathrm{dc}, i}
$$

Rather than modeling of the complete system, treating excitation coil and core as magnetic sources to determine the flux through the pickup coils, we propose to model the flux $\Phi_{\mathrm{fg}}(t)$ as being gated by an effective permeability $\tilde{\mu}(t)$. Unlike in the derivation of (1), the magnetic flux density is inhomogeneously distributed in the planar core. For each path it will therefore see a different effective permeability which becomes a smeared out and position dependent version of the original one. Using a similar expansion as in (1) results in a total voltage of

$$
V(t) \approx-\sum_{i=1}^{N} \int_{A_{i}}\left(\tilde{\mu} \dot{H}_{\mathrm{fg}}+\dot{\tilde{\mu}} H_{0}+\mu_{0} \dot{H}_{\mathrm{dc}}\right) d \mathbf{a} .
$$

The additional term that appears is a result of the direct coupling between the coils. In a symmetric Vacquier setup the inductive parts cancel to give an effective fluxgate equation for a planar assembly,

$$
V_{12}(t) \approx 2 H_{0} \sum_{i=1}^{N} \int_{A_{i}} \dot{\tilde{\mu}}(\mathbf{x}, t) d \mathbf{a}
$$

This equation describes a fluxgate effect that is highly geometry dependent, expressed through the effective permeability $\tilde{\mu}(\mathbf{x})$. As a result of gradual core saturation, the effective permeability is a smeared out version of the original one $\left(\tilde{\mu}^{\prime}(x) \leq \mu^{\prime}\right)$ which results in a loss of sensitivity. In addition, more supply current is required to satisfy condition $H_{e x} \gg H_{S}$ as a large part of the excitation energy is lost to stray fields and direct coupling. On the other hand, the planar core structure is advantageous as it requires less total excitation flux to saturate and the small core cross section allows for operation at higher frequencies when considering eddy influences. It is interesting to notice how the Vacquier setup is stable against interfering effects such as stray fields and direct couplings that must in principle also appear in 3D setups but are usually not modeled. This stability, however, requires a precisely fabricated symmetrical setup that is sensitive to mechanical tolerances.

\section{Simulation of the Effective Model}

Equation (5) demonstrates that the fluxgate effect in a Vacquier-setup is, in principle, very stable. However, the sensitivity strongly depends on the system geometry through how efficiently active flux is generated and the core is saturated. The actual quantity of interest, and the only remainder in Equation (5), is the part of the active flux that is generated by the magnetic core. For any specific setup it can be modeled by a magnetostatic FEM simulation. In Figure 2 we show FEM simulation results (ANSYS Maxwell) for a realistic setup similar to the one depicted in Figure $1 \mathrm{~b}$ with core dimensions of $10 \times 1 \times 0.1 \mathrm{~mm}^{3}$. The excitation coil is homogenized with dimensions of $5.2 \times 4.2 \times 0.1 \mathrm{~mm}^{3}$ directly and symmetrically placed below the core. The core material parameters are $\mu r=1000$ and $B S=0.5 T$ using a simple atan-model for the magnetization. The magnetic flux density generated by core is obtained by subtracting simulation results with and without core from each other. For positions within the pickup region (adjacent coil in Figure 1b), Figure 2a demonstrates how the (local) active flux density behaves as a function of the excitation current. The saturation point at $\approx 50$ AT is clearly visible. The sensitivity of the devise is proportional to the sharpness of this bend which is given by the maximum of the curvature. The latter is shown in the pickup region in Figure $2 \mathrm{~b}$. Regions in the direct vicinity of the core and close to the excitation coil 
(small values of $y$ ) contribute most to the fluxgate effect. The effective, active flux for three winding layouts is shown in c).

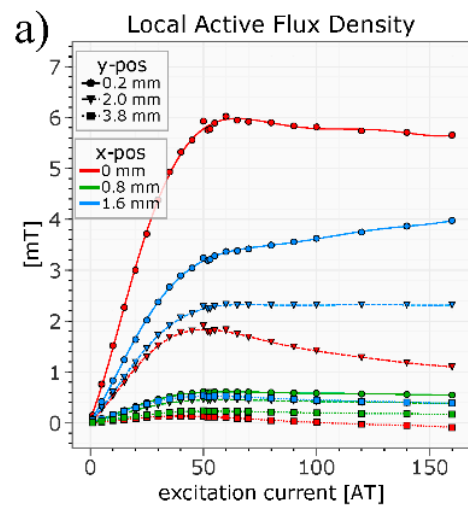

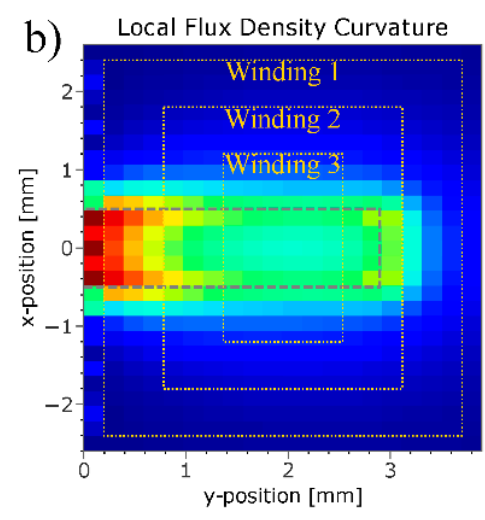

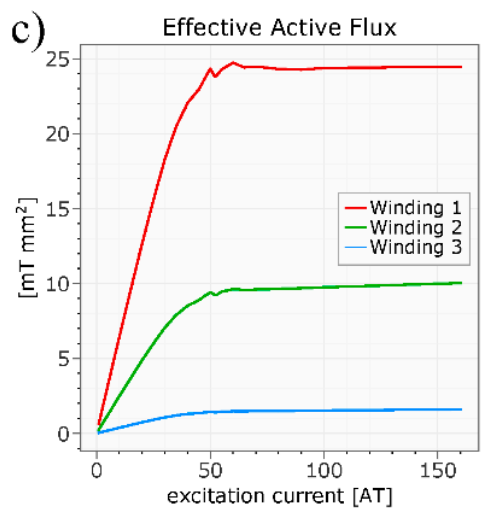

Figure 2. FEM simulation results.

The simulations show that the planar geometry and the resulting gradual core saturation modifies the original atan gating function. The active flux and the curvature (sharpness of bend) depend strongly on the system geometry, specifically on the layout of the pickup coil. The simulations suggest that adjacent coil structures are inferior to overlapping excitation and pickup coils as are used in [6].

\section{Conclusions}

The derivation of the fluxgate equation was reviewed for planar geometries. The proposed extension shows that Vacquier-setups enable a highly stable fluxgate mechanism where stray fields and direct couplings between excitation and pickup coils reduce only energy efficiency while retaining potential detectivity. The spatial dependence of the active flux, predicted by the theoretical formalism and the observed gradual core saturation, was simulated by FEM. The results can be used to test and improve planar layouts using only pure magnetostatic simulations.

Funding: This project has been supported by the COMET K1 centre ASSIC Austrian Smart Systems Integration Research Center. The COMET-Competence Centers for Excellent Technologies- Program is supported by BMVIT, BMWFW and the federal provinces of Carinthia and Styria.

Author Contributions: Simulation and theoretical model: M.O., Discussion and project setup M.O. M.L. and N.N., Paper written by M.O.

Conflicts of Interest: The authors declare no conflict of interest.

\section{References}

1. Ripka, P. Advances in fluxgate sensors. Sens. Actuators A Phys. 2003, 106, 8-14.

2. Burger, J. The theoretical output of a ring core fluxgate sensor. IEEE Trans. Mag. 1972, 8, 791-796.

3. Park, H.S.; Hwang, J.S.; Choi, W.Y.; Shim, D.S.; Na, K.W.; Choi, S.O. Development of micro-fluxgate sensors with electroplated magnetic cores for electronic compass. Sens. Actuators A Phys. 2004, 114, 224229.

4. Huang, W.S.; Lu, C.C.; Jeng, J.T. A novel 3D CMOS micro-fluxgate magnetic sensor for low magnetic field detection. Sensors, 2010 IEEE. IEEE, 2010, pp.1791-1794.

5. Vincueria, I.; Tudanca, M.; Aroca, C.; Lopez, E.; Sanchez, M.C.; Sanchez, P. Flux-gate sensor based on planar technology. IEEE Trans. Mag. 1994, 30, 5042-5045.

6. Baschirotto, A.; Dallago, E.; Malcovati, P.; Marchesi, M.; Venchi, G. Development and comparative analysis of fluxgate magnetic sensor structures in PCB technolozgy. IEEE Trans. Mag. 2006, 42, 1670-1680. 
7. Lenzhofer, M.; Ortner, M.; Schulz, G., Stahr, J. A New Approach For a Planar Miniaturized PCB Based High Sensitivity Fluxgate Sensor Design. Sensor 2017, 41-45, doi:10.5162/sensor2017/A1.4.

8. Primdahl, F. The fluxgate magnetometer. J. Phys. E Sci. Instrum. 1979, 12, 241.

(c) (1)

(C) 2018 by the authors. Licensee MDPI, Basel, Switzerland. This article is an open access article distributed under the terms and conditions of the Creative Commons Attribution (CC BY) license (http://creativecommons.org/licenses/by/4.0/). 